\title{
Mutual gaze with a robot affects human neural activity and delays decision-making processes
}

\author{
Marwen Belkaid ${ }^{1, *}$, Kyveli Kompatsiari ${ }^{1, *}$, Davide de Tommaso ${ }^{1}$, Ingrid Zablith ${ }^{1}$, and Agnieszka \\ Wykowska ${ }^{1, \S}$
}

\author{
Affiliations \\ ${ }^{1}$ Istituto Italiano di Tecnologia (IIT), Genova, Italy. \\ ${ }^{*}$ Equal contribution \\ ${ }^{\S}$ Corresponding author
}

${ }^{\S}$ Corresponding author information:

Agnieszka Wykowska

Istituto Italiano di Tecnologia

Center for Human Technologies

Via Enrico Melen, 83

16152 Genoa, Italy

\section{Email addresses}

marwen.belkaid@iit.it (MB), kyveli.kompatsiari@iit.it (KK), davide.detommaso@iit.it (DDT), ingrid.zablith@iit.it (I.Z.), agnieszka.wykowska@iit.it (AW)

\section{Abstract}

In most everyday life situations, the brain needs to engage not only in making decisions, but also in anticipating and predicting the behavior of others. In such contexts, gaze can be highly informative about others' intentions, goals and upcoming decisions. Here, we investigated whether a humanoid robot's gaze (mutual or averted) influences the way people strategically reason in a social decisionmaking context. Specifically, participants played a strategic game with the robot iCub while we measured their behavior and neural - activity by means of electroencephalography (EEG). Participants were slower to respond when iCub established mutual gaze prior to their decision, relative to averted gaze. This was associated with a higher decision threshold in the drift diffusion model and accompanied by more synchronized EEG alpha activity. In addition, we found that participants reasoned over the robot's actions in both conditions. However, those who mostly experienced the averted gaze were more likely to adopt a self-oriented strategy and their neural activity showed higher sensitivity to outcomes. Altogether, these findings suggest that robot gaze acts as a strong social signal for humans, modulating response times, decision threshold, neural synchronization, as well as choice 
strategies and sensitivity to outcomes. This has strong implications for all contexts involving humanrobot interaction, from robotics to clinical applications.

\section{Summary}

Task-irrelevant robot gaze evokes neural suppression, delays decisions, and alters social decisionmaking strategies.

\section{Introduction}

Many human decisions are made in social contexts, which often requires assessing the intentions of others. In order to infer others' mental states and to be able to predict their behavior, humans rely largely on nonverbal cues. In particular, eye contact (or mutual gaze) is a strong communicative signal in human interactions which can convey information about one's state, goals, intentions, or willingness to interact $(1,2)$. Mutual gaze has been extensively investigated in studies showing that it affected arousal level(3) and cognitive processes such as memory, attention, and motor actions(4). Eye contact is known to modulate the concurrent and/or immediately following cognitive processing (5). However, its effects on behavioral and neural responses in the context of complex social decision-making have been rarely addressed, in human-human or human-robot interaction fields of study.

Behavioral and neuroeconomics studies investigate complex social dynamics using tasks adapted from game theory. Strategic games, such as the "Chicken game” (17), are specifically designed to address social decisions in conflicting situations which may involve coordination, reciprocity, risk-taking, competition, altruism, and other mechanisms. Recently, various studies have combined strategic games with neuroimaging techniques in the quest for a better understanding of the neural mechanisms at play in social decision-making, see(6) for a review. For instance, the chicken game has been previously employed to investigate the influence of personal traits(7) or dyad familiarity(8) on participants' behavior and neural activity. However, these studies are generally screen-based and lack the ecological validity of real-time interactions. In this regard, robots offer a powerful solution for the design of controlled, yet naturalistic and embodied, interactions. Previous works in human-robot interaction have also proposed experiments based on strategic games, comparing different types of robot strategy $(9,10)$, robot embodiment $(11,12)$, payoff incentives(13), or group sizes in an intergroup competition(14). Notably, some of these studies also recorded participants' brain activity using functional magnetic resonance imaging (fMRI) while they played the game on-screen inside the scanner(11), sometimes after interacting with different robots outside the scanner(12). Nevertheless, the effect of robots' gaze and communicative behavior during the game in real-time interaction with a physically present embodied robot has remained unexplored.

Previous studies in human-robot interaction showed that similar to a human gaze, the robot's gaze could also act as a social signal and modulate the interaction. For example, eye contact improves the evaluation of the robot and of the interaction (e.g., during human-robot handovers(15), or during joint attention $(16,17)$ ), strengthens the sensitivity to robot's gaze (i.e., people perceive a robot's gaze directed towards them, but not when it is directed to a person sitting nearby)(18), and increases the 
attribution of intentionality(19) and subjective feelings of engagement(17, 19, 20). Furthermore, it can affect the quality of human-robot interaction depending on different parameters (e.g., robot's likeability and physical proximity(21), social context of the conversation(22)). Moreover, it has been shown that adding joint attention capabilities to a robot can facilitate participants' performance in a human-robot task $(15,23)$. The majority of the abovementioned studies used subjective reports or focused on the perceived quality of interaction. Despite the valuable insights offered by subjective reports, explicit measures cannot always target specific cognitive mechanisms that occur in real-time interactions, which are often implicit and beyond conscious awareness. Recently, studies using objective measures revealed that eye contact with iCub humanoid robot elicited a higher degree of attentional engagement (i.e., longer fixations to the face of the robot during eye contact condition)(24). Additionally, initiating or being exposed to eye contact with a humanoid robot modulated humans' oscillatory brain activity in the same frequency range as in the case of human eye contact $(25,26)$ (i.e. alpha frequency range).

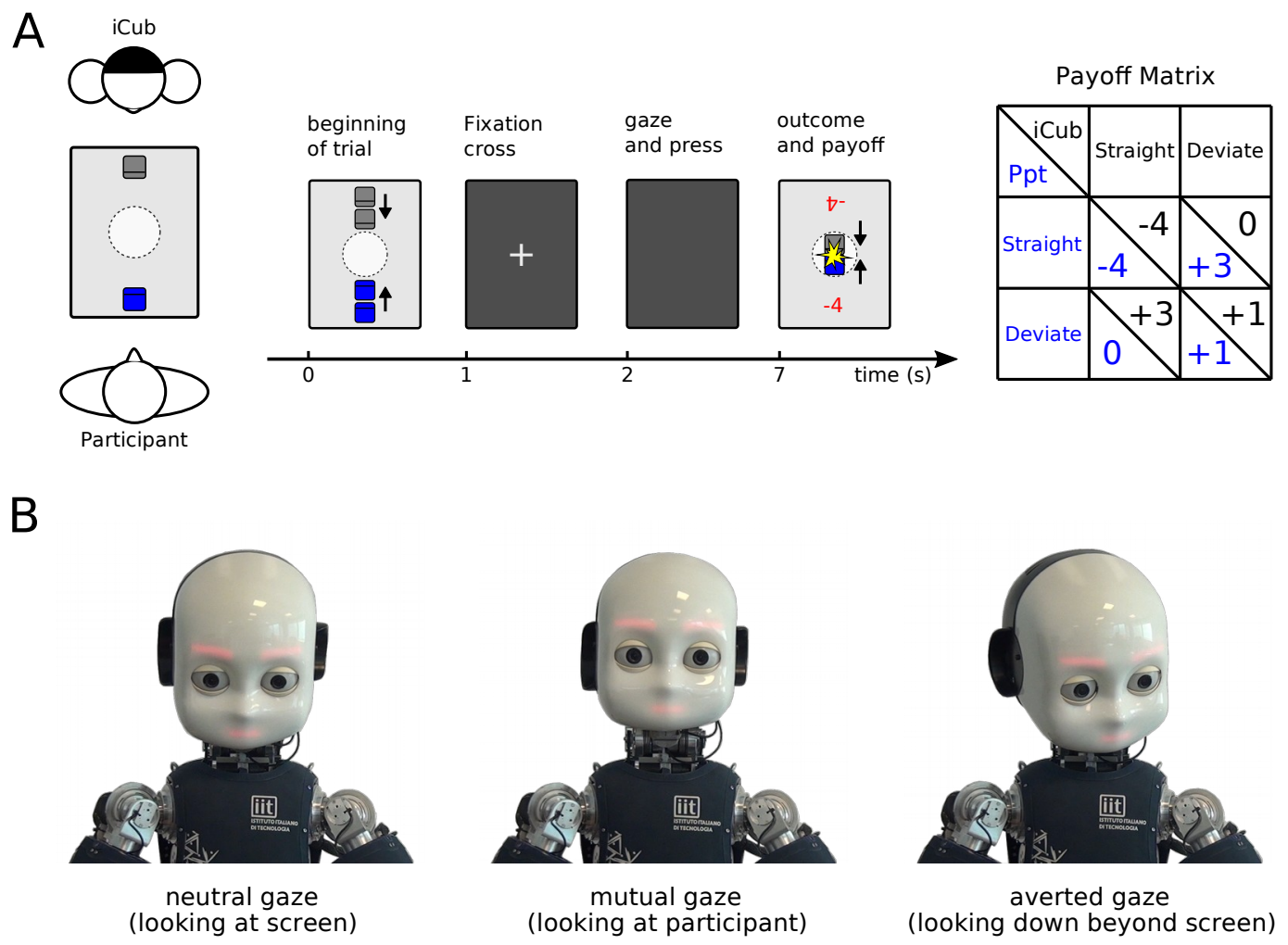

Figure 1: Human-robot Chicken game. A) Schematic illustration of the experiment, trial structure and payoff matrix. Participants sat facing the iCub robot while the game was displayed on screen placed between them. The players played an adaptation of the Chicken game in which we manipulated the robot gaze during the decision period (labeled "gaze and press", 2-7 s). After looking at iCub, participants had to decide whether to go straight or deviate. The outcome of the trials and each player's payoff was determined by the combination of the two players' choices. B) During the entire trial, except for the decision period, iCub looked at the screen (neutral gaze). During the decision step, iCub either established or avoided eye contact with the participant (mutual or averted gaze, respectively). 
In this paper, we present a study of human decision-making under the influence of the communicative behavior (gaze in this case) exhibited by a humanoid robot in an interactive setup employing behavioral and neuroscience measures. More precisely, we examine the influence of mutual versus averted gaze on participants' behavior and neural activity, measured by electroencephalography (EEG), while they play the Chicken game - a strategic game which depicts a situation in which two drivers of simulated cars move towards each other on a collision course and the outcome depends on whether the players yield or keep going straight (28) - against the iCub robot(27). Based on the literature on human-human interaction $(4,5)$ we hypothesize that, mutual gaze should engage more cognitive processes, relative to averted gaze. Such effect could be related to a higher degree of mentalizing (to anticipate iCub's action based on its gaze) or to suppression of irrelevant salient signal (to make decisions independently from iCub's gaze). Therefore, we expected this would be reflected in behavioral measures (longer response times), EEG signal (alpha synchronization), and choice sequences and strategies.

\section{Implementation of the Human-robot Chicken game}

Participants sat in front of the iCub robot while the game was displayed on a screen placed horizontally on a table between them and the robot (Fig 1A). The experiment consisted of an adaptation of the Chicken game(28). In the beginning of every round (hereafter "trial”), two car images, one per player, were shown on each side of the screen. The cars started moving toward each other and stopped halfway before reaching the center. Then, the screen turned black and a fixation cross was shown for 1 second. After fixation cross disappeared, the screen remained black for 5 seconds and both players had to choose their move between going straight or deviating. Critically, during this period, participants were instructed to look at the robot, which either looked directly at the participant (hereafter labeled Mutual gaze) or avoided eye contact by looking to the side (hereafter labeled Averted gaze; see Fig 1B). For the reasons why averted gaze has been chosen as comparison for the mutual gaze, see (29). Both players had an occluder covering their response buttons, thereby preventing the opponent from anticipating their choices. At the end of the trial, the cars appeared on the screen again and displayed the action selected by the players. The possible outcomes were: i) both go straight and crash, resulting in the highest loss for both, ii) both deviate, obtaining the highest joint payoff, iii) only one goes straight obtaining the highest individual payoff. The points obtained (or lost) in the current trial were shown on each player's side of the screen (see Fig 1A for the payoff matrix). Moreover, to maintain participants' engagement, iCub verbally reacted to the outcome in $40 \%$ of the trials within each block by randomly selecting one of the predefined utterances associated with that outcome (see Table S1).

Apart from the decision period when the robot's gaze was manipulated (mutual or averted gaze), for the remaining time of the trial, the robot was looking at the screen, making periodic random saccades within that visual area. In this experiment, participants completed 250 trials divided in 5 blocks. They were assigned to either the group with $70 \%$ Mutual or $70 \%$ Averted conditions, meaning that in each block, iCub performed the type of gaze corresponding to the condition (e.g., mutual gaze in the 70\% Mutual condition) in $70 \%$ of the trials within each block in a pseudo-randomized manner. The other type of gaze (e.g. averted gaze in the $70 \%$ Mutual condition) was performed in the remaining $30 \%$ of

the trials. With this manipulation, we sought to investigate how participants' behavior could be influenced by establishing mutual gaze with the robot prior to decision-making (within-participants) 
and the effect of the degree of exposure to mutual versus averted gaze on their overall strategy in the game (between-participants).

Participants were told that their objective was to maximize their total score regardless of the robot's score. This was to make the always-go-straight strategy less appealing, because as much as it guarantees a higher score than the opponent, this strategy can lead to very low negative scores due to numerous crashes. Moreover, iCub followed a win-stay-lose-shift strategy (WSLS)(30)with a probability of $80 \%$; meaning that it was most likely to repeat an action (i.e. stay) if it had led to a positive outcome in the previous trial and do the other action (i.e. shift) otherwise. Thereby, the robot's sequence of choices had a certain structure (WSLS) that participants could capture without it being too obvious. Altogether, we gave both the incentive and the opportunity to participants to reason about the robot's actions during the experiment.

\section{Results}

\section{Performance in the human-robot Chicken game}

The “optimal”(31) (predict-opponent) and “riskiest” (always-go-straight) strategies were simulated to provide a reference point for good and bad performance respectively, see (25) for definition of the “optimal” strategy in this game. Participants' scores were markedly higher than the average score obtained when we simulated the risky always-go-straight strategy (Fig 2A). In fact, $45 \%$ of the participants obtained a positive total score and $55 \%$ obtained a higher score than iCub. However, total scores did not differ between conditions (70\% Mutual versus 70\% Averted, $t(19)=0.50, p=0.61, t$-test; Fig 2A). In addition, total scores were much lower than the average score obtained by simulation of the optimal strategy which consists in choosing the best action assuming that the opponent is using the WSLS strategy (Fig 2A). Indeed, a custom-made post-hoc questionnaire showed that $25 \%$ of the participants thought that iCub had a strategy and only $12.5 \%$ identified the actual WSLS strategy. These results are in line with those of pilot study we conducted prior to this experiment (see Materials and Methods and Supplementary Materials).

\section{Increased reaction times and decision threshold following mutual gaze}

Using a two-way ANOVA with gaze condition within- and between-subject as factors, we found no significant main effect, all $\mathrm{Fs}<1$, nor interaction, $\mathrm{F}=1.46, \mathrm{p}=0.24$, in the frequency of selected actions (Fig 2B). This means that the robot's gaze did not influence participant's subsequent choices, nor did the overall degree of exposure to the two gaze types. However, the same analysis showed a significant main effect of iCub's gaze on participants' mean response times, $F=35.11, p<0.001, \eta \quad{ }^{2}=0.034$, independent of the group they were assigned to (70\% Mutual or 70\% Averted). More specifically, participants responded faster following an averted gaze than following a mutual gaze (in 70\% Mutual, $\mathrm{M}_{\text {averted }}=1993 \mathrm{~ms}, \mathrm{SEM}=106, \mathrm{M}_{\text {mutual }}=2117$, SEM=99; in 70\% Averted, Merted $=2086$, SEM=180, $\mathrm{M}_{\text {mutual }}=2256$, SEM=174; Fig 2C). This within-subject effect of gaze was also revealed by our pilot study where participants were exposed to the same amount of mutual and averted gaze instances (see Materials and Methods for procedure description and Supp Figure 1A for results). Thus, in this task, 
mutual gaze consistently elicited longer response times compared to averted gaze, whether the former was predominant, equally frequent or more occasional than the latter.

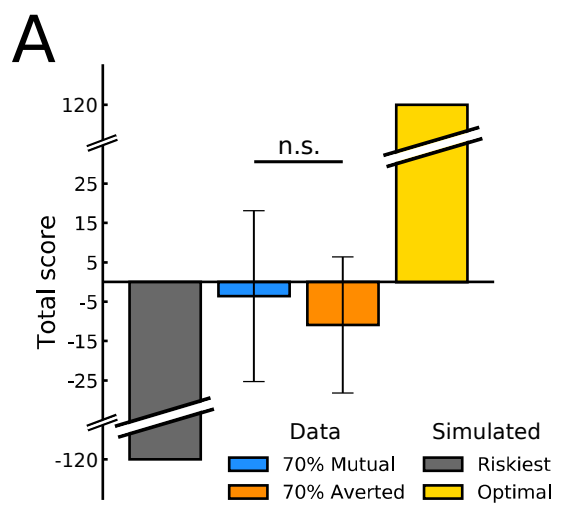

D

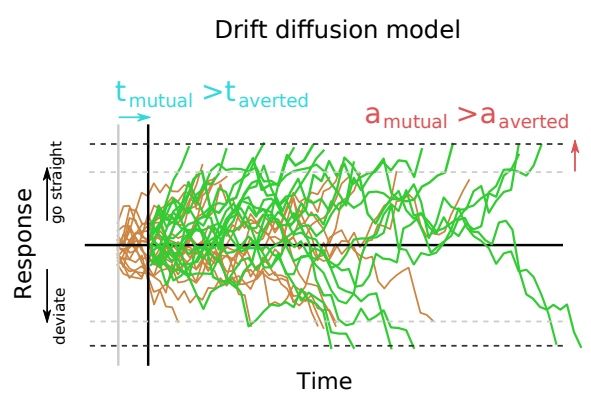

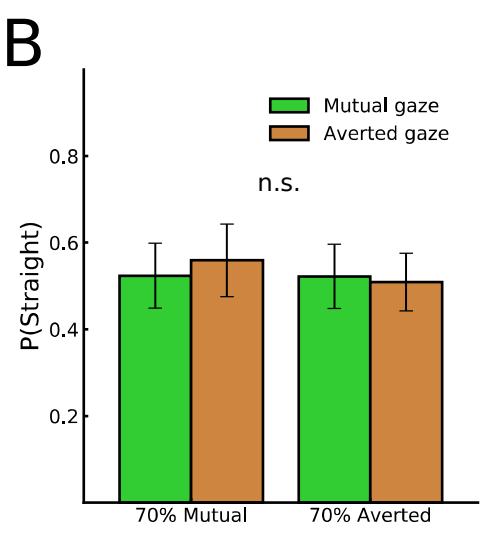

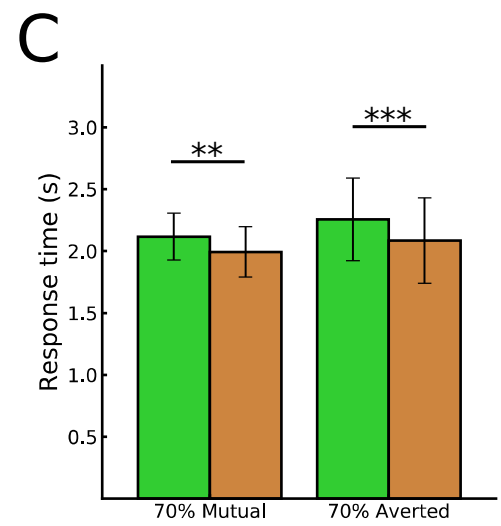

$\mathrm{E}$

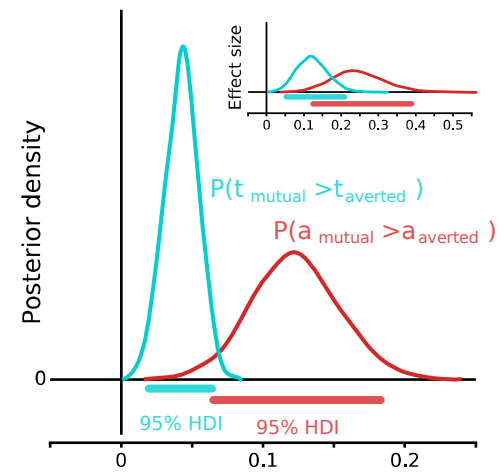

Figure 2: Participants' performance and response times. A) Participants' total scores did not differ between conditions. Average performance from simulations of two extreme strategies are provided for comparison (see text). B) No main effects or interaction of the condition or gaze type was found on the proportion of "straight" choices. C) The gaze type (within-subject) had a significant main effect on participants' response times. D) The drift diffusion model describes decisions as a noisy drift process where an action is selected when the corresponding boundary is crossed. Five variants were tested assuming that the robot's gaze had an effect of one, two or none of the model parameters. Upon Bayesian parameter estimation, the best fitting model was found to be the one imputing the difference in response times to an effect on both the non-decision time $t$ and the decision threshold $a$. DIC: deviance information criterion. BPIC: Bayesian predictive information criterion. Lower values are better. E) Posterior density of the effect distributions for the best fitting model showing significant effects on both $a$ and $t$ parameters. HDI: highest density interval. Inset, effect sizes (see Materials and Methods). Error bars represent 95\% confidence intervals. ${ }^{* *} \mathrm{p}<0.01,{ }^{* * *} \mathrm{p}<0.001$. n.s., not significant at $\mathrm{p}>0.05$.

In line with our hypothesis, the delayed responses within-subjects following mutual gaze may suggest that mutual gaze entailed a higher cognitive effort, for example by eliciting more reasoning about iCub's choices or higher degree of suppression of the (potentially distracting) gaze stimulus, which was irrelevant to the task. To further investigate this hypothesis, we analyzed participants' choices with the drift diffusion model(32). This widely used computational model assumes that decisions arise from 
relative evidence accumulation over time in favor of one of two alternatives (see illustration in Fig 2D). The main parameters include the drift rate $v$ (rate of evidence accumulation), decision boundary $a$ (threshold to be reached to make a decision) and non-decision time $t$ (dedicated to stimulus encoding and motor execution, for example). By fitting both alternatives' selection rates and response time distributions with this model, we sought to infer the latent psychological processes explaining the delayed responses following mutual gaze. Specifically, a longer and more effortful reasoning process predicts an effect on decision threshold(32). Using hierarchical Bayesian parameter estimation (see Materials and Methods), we compared the fitness of different models assuming that the robot's gaze had an effect on either the drift rate, the non-decision time or the decision boundary. Based on the deviance information criterion (DIC) and the Bayesian predictive information criterion (BPIC), we found that the two best fitting models were those presuming an effect on non-decision time or decision threshold: a difference in non-decision time explained the data slightly better in the main experiment (Fig 2D) whereas a difference in decision threshold explained the data better in the pilot study (Supp Fig 1C). Therefore, we also tested a model imputing the response delay to both non-decision time and decision threshold. This model fitted the data better than the others in both datasets (Fig 2D and Supp Fig 1C). In the main experiment, both the decision threshold $a$ and the non-decision time $t$ were significantly higher following mutual gaze compared to averted gaze $(\mathrm{P}(\Delta a>0)=1.0, \mathrm{P}(\Delta t>0)=1.0)$ with a larger effect size for the former (Fig 2E). However, in the pilot study, only the difference in decision threshold reached significance $(\mathrm{P}(\Delta a>0)=0.99, \mathrm{P}(\Delta t>0)=0.87$; Supp Fig 1D). Taken together, these results show that the effect of gaze on response time is mainly driven by an effect of the decision threshold. This suggests a longer and more effortful decision process in case of mutual gaze, possibly due to the engagement of mentalizing processes to try to interpret the robot's intention or of suppression mechanisms to make decisions independently from (potentially distracting) iCub’s gaze.

\section{More synchronized alpha rhythm during mutual gaze}

Behavioral analysis during decision period revealed a clear effect of iCub’s gaze on participants' response time. We investigated whether this effect would also translate into distinct neural responses. For instance, time-frequency analysis can reveal different oscillatory activities across time in the human brain's prominent rhythms that are linked to distinct neural processes (e.g., see(33)). Therefore, we analyzed participants' brain activity in the time period between the completion of the robot gaze movement (eyes and neck) and the average response time. We focused on theta and alpha bands which have been shown to be modulated by decision-making $(34,35)$, attention $(33,36)$ and eye contact $(26$, $37,38)$.

We did not observe any significant differences in theta frequency range. However, we found that participants responded with a higher alpha synchronization during mutual gaze compared to averted gaze in a parietal area (corrected for multiple comparisons $\mathrm{p}=.03$; see Materials and Methods) during the time window 0.31-0.95 s relative to the initiation of robot's head movement toward the gaze direction (see Figure 3). To locate the specific cluster of electrodes, we further averaged the data across the entire temporal period and compared the spatial data across conditions using the same analysis as above. Results showed that the parietal cluster consisted of six electrodes Pz, P3, P1, PO3, POz, P2. The averaged alpha values were lower in averted gaze $\mathrm{M}=131.02$, SEM = 34.34 compared to mutual gaze $\mathrm{M}=192.13$, SEM = 52.55. Increase in alpha power has been associated with brain processes 
related to the suppressing function of attention, i.e., suppressing cortical activity related to distractors or irrelevant information(39, 40), for a review see(33). Higher alpha synchronization in mutual gaze might indicate an increased need to suppress distraction related to the gaze exhibited by the robot, while focusing on task-relevant information. This results in longer reaction times for mutual, relative to averted gaze, trials.
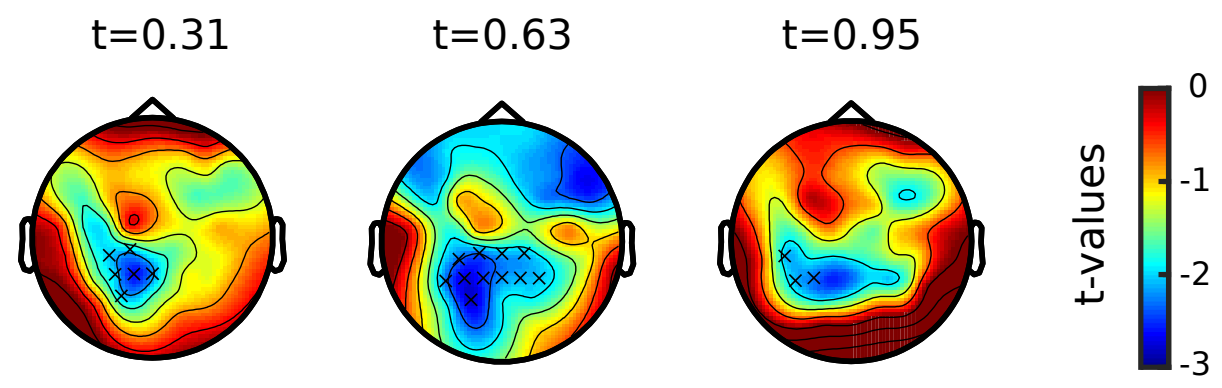

Figure 3: Scalp topographies between gaze types during decision period. Topographies show $t$-values maps of the difference in elicited alpha oscillations ( $8-12 \mathrm{~Hz}$ ) between gaze conditions (averted gaze - mutual gaze) considering data from both participant groups. Statistically significant clusters are marked by x crosses. Differences between conditions were found by non-parametric cluster-based permutation tests. $t$-values are defined as the ratio of the difference between the estimated mean values of two conditions to its standard error. The topographies are depicted in a time range of $\mathrm{t}=0.31 \mathrm{~s}$ to $\mathrm{t}=0.95 \mathrm{~s}$, relative to the establishment of the exhibited gaze (every $320 \mathrm{~ms}$ ).

\section{Reasoning about the robot's actions throughout the game}

In order to assess how much participants reasoned about the robot's choices during the game, we simulated three computational models taken from the literature(41). These models make value-based decisions and compute different types of prediction errors to update the choices values over time (Fig 4A; see Materials and Methods for implementation details). The first model is a classical reinforcement learning model in which the action that was most rewarded in the recent past is more likely to be repeated(42). The second model attempts to predict the opponent's decision by estimating the probability that they select an action based on their recent history of choices. The model then selects the action which maximizes the gain (or minimizes the loss) based on the payoff matrix(41). The third model builds on the second model and incorporates the influence of the player's own recent choices on the opponent's decisions by assuming that the other is tracking the player's probabilities of choices too(41). These models thus describe three levels of reasoning about the other (i.e. mentalizing). The first model derives decisions solely from recent rewards (level 0 ), the second builds predictions of the opponent's choices based on the recent history (level 1) and the third does so by also assuming a model of the other's strategy (level 2).

Upon optimization, we found that participants choice sequences altogether were best reproduced by the model with the highest level of mentalizing ( $\log (\mathrm{L})$ across models, $H=23.64, p<0.0001$, KruskalWallis test; Inf versus Fic, $U=433.0, p=0.0002$, Fic versus RL, $U=547.0, p=0.007$, Mann-Whitney 
tests; Fig 4B). By replicating previous findings in human-human strategic games(41), we demonstrated that participants reasoned about iCub's actions in a way that is similar to settings involving human opponents. Nevertheless, examining the best fitting model for each participant individually revealed little difference between our experimental conditions. In other words, participants exposed to $70 \%$ of mutual or averted gaze were fitted in similar proportions by the models (Fig 4C). Moreover, we examined the percentage of choices that were correctly predicted by the best fitting model for each participant. Results showed no significant effect of the trial's gaze type within each condition (in 70\% Mutual, $U=171.5, p=0.22$; in 70\% Averted, $U=192.0$, $p=0.41$, Mann-Whitney test; Fig 4D). Overall, while suggesting that participants reasoned about the robot's actions during the game, these computational models were unable to capture subtle differences which could further explain the effect of the mutual gaze on participants' behavior.
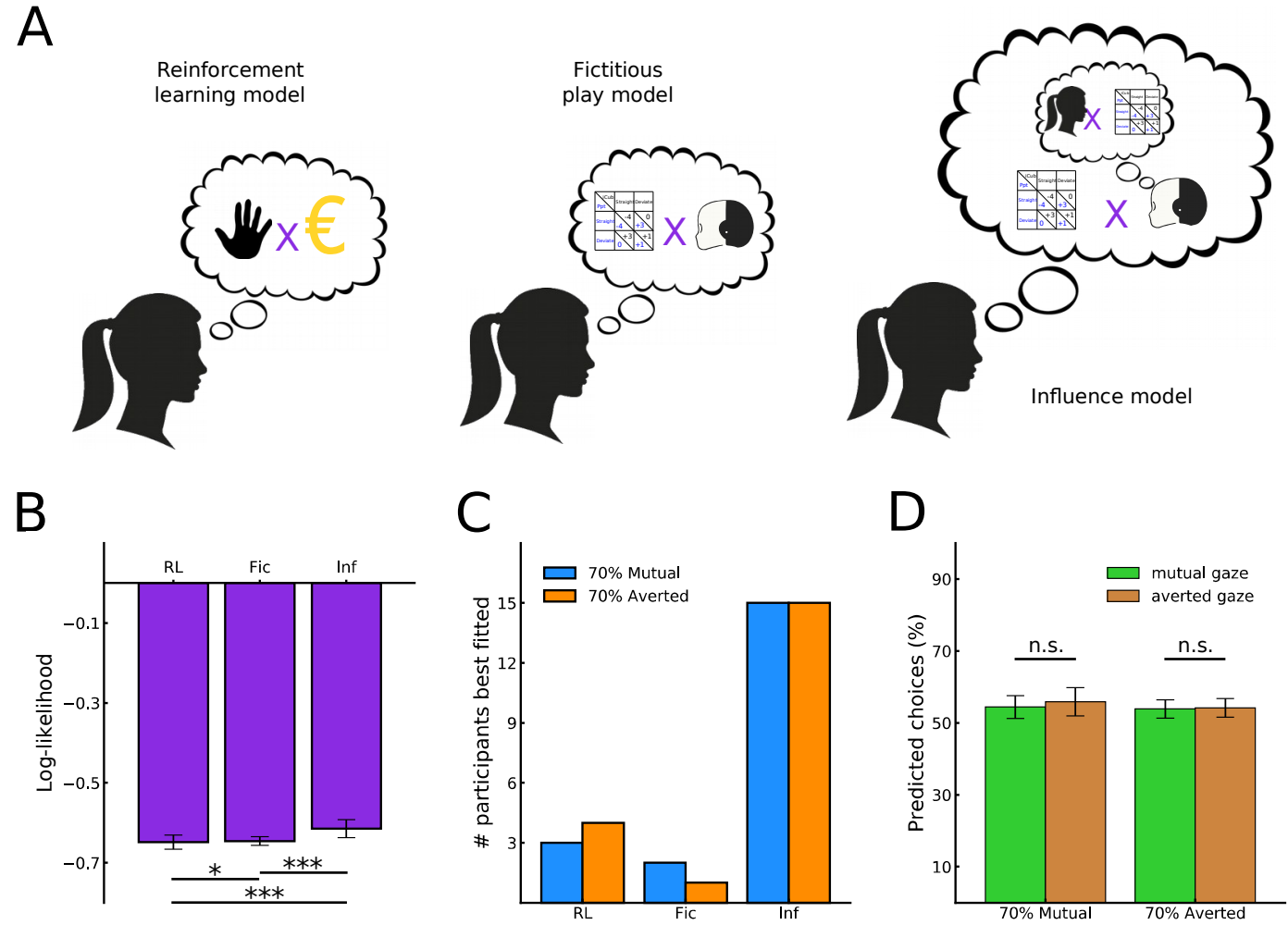

Figure 4: Computational models of participants' degree of reasoning about iCub's actions. A) Schematic illustration of the models. The reinforcement learning model (RL) makes decisions based on the recently selected actions and their outcome. The fictitious play model (Fic) makes decisions based on the game's payoff matrix and the predicted action of the opponent. The influence model (Inf) does the same while assuming the opponent is also predicting the player's choices and incorporating the influence of its own actions in its predictions of the opponent's decisions. B) The overall log-likelihood of the influence model fitted to participants' choices is significantly greater than the two other models, suggesting a high level of reasoning about iCub during the game. C) Considering the best fitting model for each participant individually did not reveal a strong difference between those exposed more to one type of gaze compared to the other. D) The percentage of trial-by-trial choices predicted by the model was similar following a mutual or averted gaze. Error bars represent $95 \%$ confidence intervals. $* \mathrm{p}<0.05$, ${ }^{* * *} \mathrm{p}<0.001$. n.s., not significant at $\mathrm{p}>0.05$. 


\section{More self-oriented strategic patterns under higher exposure to averted gaze}

To further investigate the influence of iCub's gaze on participants' decisions, we analyzed the frequency of occurrence of three patterns of strategic behavior in their choice sequences which could characterize the extent to which they incorporated information about their opponent in their decisions. The first pattern is 'win-stay-lose-shift' (WSLS), which is a typical decision-making strategy. It consists in repeating the previous action if it led to a positive outcome and abandoning it otherwise. This strategy is self-oriented, in the sense that it only requires information about the player's own actions and outcome history and can be employed even in individual decision-making contexts. As a reminder, this was iCub's strategy in $80 \%$ of the trials.

The second pattern that we examined is classical strategy in game theory called 'tit-for-tat' (T4T). The idea here is to reproduce the opponent's previous choice. This strategy is known to be efficient in strategic games as it dissuades opponents from attacking and encourages them to cooperate by favoring actions which generate mutual gains. Additionally, we added another pattern labeled 'stay-shiftimitation' (SS-Imit). In this strategy, the player copies the opponent's behavior in repeating the last trial's choice or changing. In contrast to the first strategy, the two latter are other-oriented since they process information about the opponent's history of actions and outcomes.
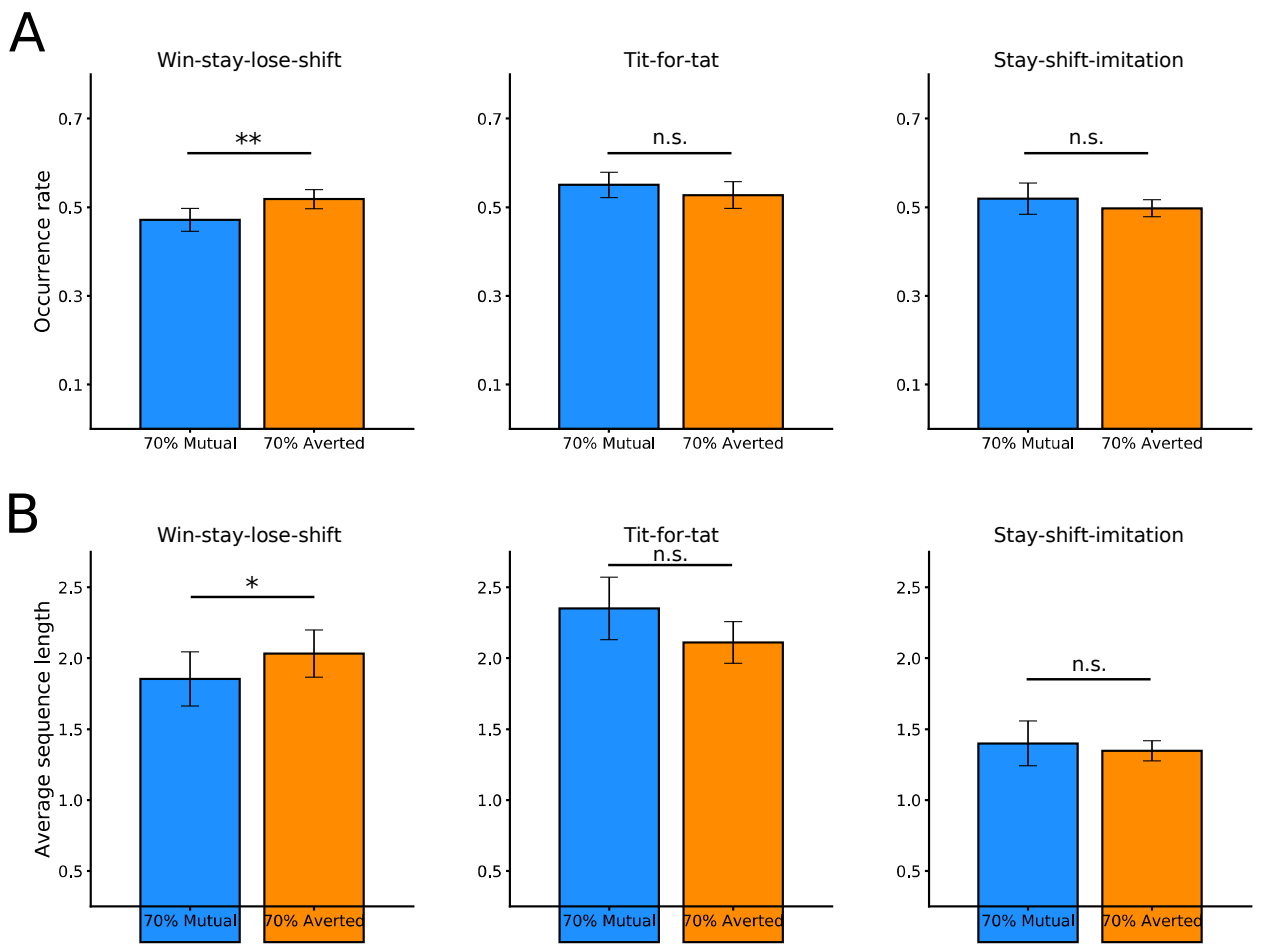

Figure 5: Patterns of self-oriented and other-oriented strategic behaviors in participants' choice sequences. A) Occurrence rates of one self-oriented (win-stay-lose-shift, WSLS) and two other-oriented (tit-for-tat, T4T; stay-shiftimitation, SS-Imit) patterns (see text for detailed description). Self-oriented WSLS appeared significantly less in the choices of participant who were more exposed to the mutual gaze. No significant difference was found for T4T and SS-Imit. B) The average length of sequences of the WSLS pattern was significantly greater in the $70 \%$ Averted condition. No significant difference was found for T4T and SS-Imit. Error bars represent 95\% confidence intervals. * $\mathrm{p}<0.05,{ }^{* *} \mathrm{p}<0.01$. n.s., not significant at $\mathrm{p}>0.05$. 
We analyzed participants' choice sequences trial-by-trial looking for occurrences of these patterns of strategic behavior. Results showed that the pattern WSLS was found more frequently in the sequences of participants of the $70 \%$ Averted condition compared to 70\% Mutual ( $U=108.5, p=0.006$, MannWhitney test; Fig 5A). In other words, the occurrence rate of the self-oriented pattern was significantly lower under higher exposure to the mutual gaze. Interestingly, considering data from a pilot study, we found that when participants were equally exposed to both types of gaze, the occurrence rates of WSLS was similar to the $70 \%$ Mutual condition and also contrasted significantly with the $70 \%$ Averted condition (see Supplementary materials and Supp fig 3A). However, the difference between conditions for T4T and SS-Imit was not significant (T4T, $t(19)=-1.07, p=0.28$, SS-Imit, $t(19)=-1.02, p=0.30, t$ test; Fig 5A). Furthermore, we computed the average length of sequences of the strategic patterns to test for their recurrence over several trials. Again, we found a difference between the $70 \%$ Mutual and $70 \%$ Averted conditions for the WSLS pattern ( $U=120.0$, $p=0.015$, Mann-Whitney test; Fig 5B) with longer sequences in the latter condition. On the other hand, the average length of sequences of the other patterns did not differ significantly between conditions (T4T, $U=150.5, p=0.092$, SS-Imit, $U=189.0$, $p=0.38$, Mann-Whitney test; Fig 5B). In summary, this analysis suggests that the likelihood of adopting self-oriented strategy was higher among those who were exposed mainly to averted gaze, relative to those who were exposed mainly or equally to mutual gaze.

\section{EEG markers of performance monitoring modulated by gaze: exploratory analyses}

Because participants exhibited different strategic patterns depending on the degree of exposure to mutual or averted gaze, we reasoned that they would process their outcomes distinctly, as the analyzed strategies are inherently related to processing outcome and performance. At the neural level, performance and outcome monitoring are typically reflected in event-related potentials (ERPs) of the EEG signal, such as feedback-related negativity (FRN), error-related negativity (ERN), Reward Positivity (RewP), and the positive component P2 preceding FRN (43-47). Therefore, we examined ERPs during the period when participants had access to information about the outcome of the trial, i.e., within the broad time window from the offset of the black screen (the moment when the cars start moving after the action decisions have taken place) to the period after the score was presented (from 0 to 2000 ms after onset of the animations; see Fig 1A). However, as our task was a dynamic game, with information about performance becoming available only gradually, the observed ERPs might not map onto classical ERP components reported in literature. Therefore, we refrained from typical componentbased interpretations and focused on how activity evolved over time around maximum/minimum peak amplitude across consecutive 400-ms segments (see Supplementary Materials for details about the procedure). Moreover, because outcome-related brain responses often differ between positive and negative outcomes $(43,46,48)$, we submitted the averaged activity around the extracted peaks to a repeated-measures ANOVA with outcome type (win, lose) as a within-subjects factor, and gaze group (70\% Mutual gaze, 70\% Averted gaze) as a between-subjects factor. For conciseness, we only report the significant results of this analysis. 


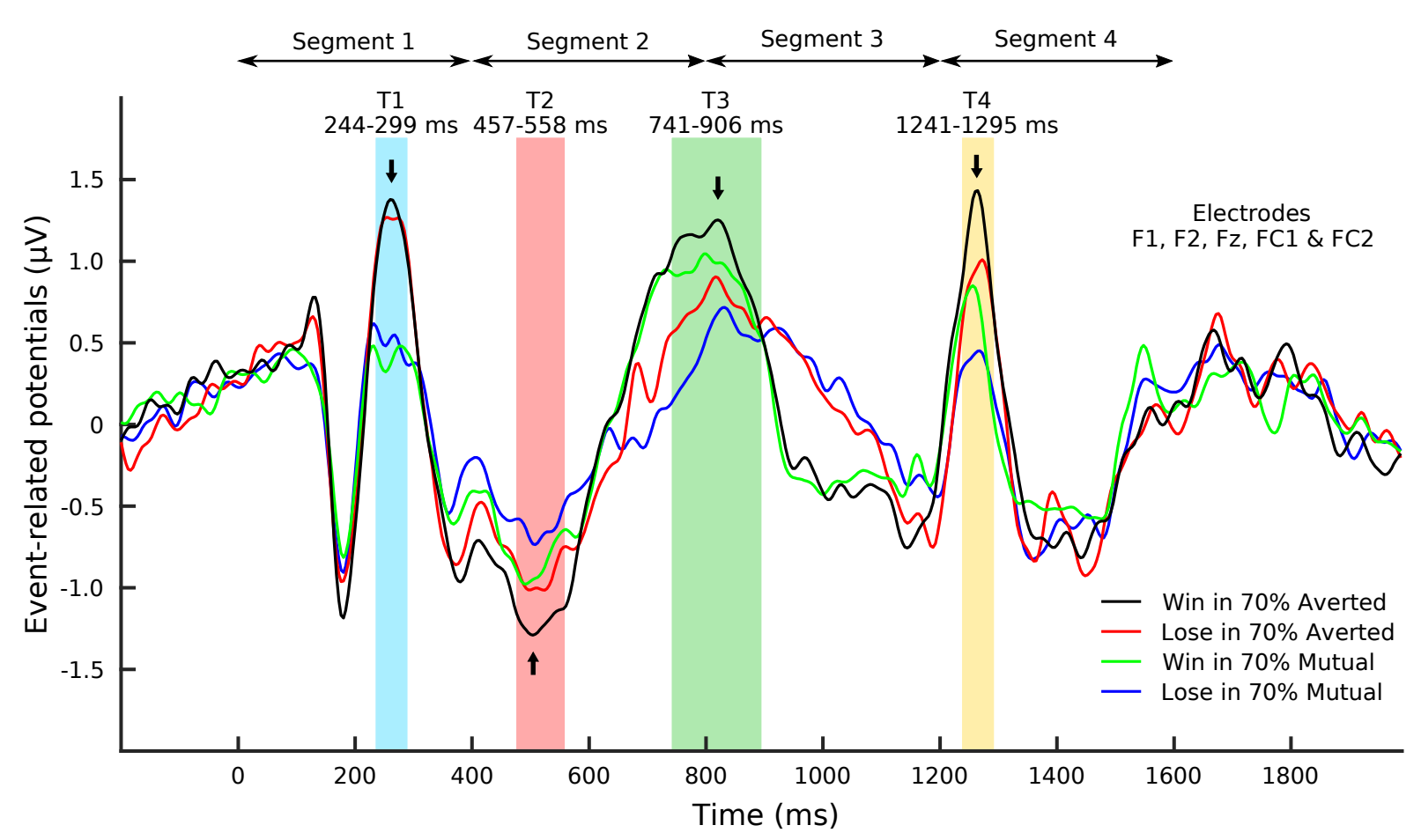

Figure 6: Grand-averaged ERP waveforms time-locked $(\mathrm{t}=0)$ to onset of feedback animation. The average is performed over F1, F2, Fz, CP1, CP2 electrodes on data from participants in the 70\% Mutual and 70\% Averted groups split into win ( +1 or +3 points) or lose ( 0 or -4 points) trials. Activity over time around maximum/minimum peak amplitude across consecutive 400-ms segments showed differentiated responses to gaze condition in the first time window, then to outcomes in the following two time windows and finally to both gaze and outcomes. Division in segments: Segment 1: $\mathrm{t}=$ 0-400 ms; Segment 2: $\mathrm{t}=400-800 \mathrm{~ms}$; Segment 3: $\mathrm{t}=$ 800-1200 ms; Segment 4: $\mathrm{t}=$ 1200-1600 ms. Time windows of analyses (Tn) within each segment: Within Segment 1: T1 = 244-299 ms (light blue box) 10\% around positive peak at $272 \mathrm{~ms}$ (black arrow); Within Segment 2: T2 = 457-558 ms (orange box), 10\% around the negative peak at $508 \mathrm{~ms}$ (black arrow); Within Segment 3: T3 = 741-906 ms (green box), 10\% around positive peak at $824 \mathrm{~ms}$ (black arrow); Within Segment 4: T4 =1241-1295 ms (yellow box), 10\% around the positive peak at $1268 \mathrm{~ms}$ (black arrow). Onset of score presentation: $1000 \mathrm{~ms}$.

Our analyses showed that at first, exposure to mainly mutual or mainly averted gaze affected the EEG activity (Figure 6, T1, blue box), modulating the first positive peak of the entire sequence, with larger positivity $\mathrm{M}=1.16$, $\mathrm{SEM}=0.27$ for group with mostly averted gaze, compared to participants mostly exposed to mutual gaze (main effect of gaze), $\mathrm{M}=0.43$, $\mathrm{SEM}=0.20, \mathrm{~F}(1,37)=4.44, \mathrm{p}=.042$, np ${ }^{2}=$ 0.1 . In a subsequent time window, there was no effect of gaze, but the EEG activity differed with respect to the outcome of participants' decisions (main effect of outcome type) (Figure 6, T2, pink box). In particular, "winning” animations elicited a larger negative deflection, $\mathrm{M}=-1.01, \mathrm{SEM}=0.12$ compared to the "losing" animations, $\mathrm{M}=-0.78$, SEM $=0.1, \mathrm{~F}(1,37)=12.17$, $\mathrm{p}=.001$, np ${ }^{2}=0.25$. This was then followed by another positive peak (Figure 6, T3, light green box) which also showed that outcome (but not gaze) modulated activity in this time period, with "winning” animations showing a larger positivity, $\mathrm{M}=0.92$, SEM = 0.09 compared to the "losing" animations (main effect of outcome 
type), $\mathrm{M}=0.6, \mathrm{SEM}=0.07, \mathrm{~F}(1,37)=17.19, \mathrm{p}<.001$, np ${ }^{2}=0.32$. Last, in the segment following the presentation of the score (Fig 6, T4, yellow box), the positive peak was modulated both by gaze and feedback, but independently of each other (main effects of outcome type and gaze but no interaction effect). Participants mostly exposed to averted gaze showed a larger positive component $\mathrm{M}=0.98$, $\mathrm{SEM}=0.21$ compared to participants mostly exposed to mutual gaze, $\mathrm{M}=0.48$, $\mathrm{SEM}=0.16, \mathrm{~F}(1,37)$ $=4.6, \mathrm{p}=.038, \mathrm{np}^{2}=0.11$. In parallel, winning feedback points showed a larger positivity, $\mathrm{M}=0.82$, $\mathrm{SEM}=0.13$ compared to the losing feedback points, $\mathrm{M}=0.6, \mathrm{SEM}=0.15, \mathrm{~F}(1,37)=3.8, \mathrm{p}=0.06$, $\mathrm{np}^{2}=0.32$.

This pattern of results on how ERPs unfolded over the critical period of time shows that the brain was initially affected by gaze: participants who were mainly exposed to avoiding gaze showed larger positivity. This is perhaps an after-effect of the preceding period where the two gaze types were displayed by the robot. However, this initial influence of gaze was in the later time windows overridden by the influence of the unfolding outcome of participants' decisions, as participants observed the car animations and realized whether they have lost or won. In this time period, winning trials evoked a more pronounced deflection (larger positivity or negativity, dependent on whether the component was positive or negative), relative to losing trials. This clearly shows that at this point, the brain differentiated the wins from losses, and this was overriding the effect of gaze. Interestingly, though, upon receiving the scores, participants' neural activity was affected both by feedback and by gaze condition. In other words, later stages of processing of performance (information about performance ultimately confirmed by the presented score) was modulated by the type of robot gaze to which participants were most exposed throughout the experiment.

\section{Discussion}

Few studies have investigated the effect of social cues on human decision-making in naturalistic interactions. Our study involved a humanoid robot, iCub, to allow us to overcome some of the methodological challenges of naturalistic interactive scenarios and enabled a real-time controlled interaction with a physically present and embodied agent. Thus, we could shed light on the influence of others' communicative signals (gaze in this case) on individuals' behavioral and neural responses in strategic interactions. Additionally, it highlights the importance of cautiously designing robots' social behavior in any context involving human-robot interactions.

In our experiment, participants played an adaptation of the Chicken game with iCub while the robot established or avoided eye contact with them prior to decision. Our results showed changes in participants' behavior depending on iCub’s gaze. First, we observed that mutual gaze with the robot delayed participants' responses. This difference in reaction times arose from a higher decision threshold in the mutual gaze condition compared to the averted gaze, thereby suggesting a longer and more effortful focus on the task. This behavioral effect was paralleled by a differential effect in synchronized alpha activity during the period of eye contact, with higher alpha synchronization compared to averted gaze. Alpha synchronization has been interpreted in literature as a marker of increased need for suppression of distractor information, for a review see(33). In our experiment, iCub's gaze was totally independent from its action and could thereby be considered by our participants as a distractor once the dissociation was detected. Therefore, our findings suggest that mutual gaze required stronger suppression, which resulted in longer reaction times and higher decision threshold. 
In addition to within-participants effects across different trials, we also analyzed strategic processes across the entire experiment, dependent on whether participants belonged to the group which was mostly exposed to the mutual gaze or to the avoiding gaze condition. We observed that while the type of gaze that participants mostly saw had no influence on choice frequencies or overall performance, patterns of strategic behaviors in participants' choice sequence differed between conditions. Indeed, when exposed more to averted gaze, participants exhibited self-oriented patterns of choices more frequently. This result suggests that higher exposure to averted gaze makes participants disengage more easily from the social interaction and rely more on self-centered information to make their decisions.

In search for the neural trace of performance/outcome monitoring processes, which would presumably underlie the different likelihood of adopting self-oriented strategy during the task, we examined ERPs of the EEG signal in the entire epoch when participants could monitor the outcome of their action decision. The results showed that the brain was indeed differentially processing information about winning and losing trials as this information was becoming available (as the car trajectories were gradually unfolding). Most importantly, being exposed mostly to mutual gaze or mostly to avoiding gaze affected the way the brain processed feedback. The group of participants who were exposed mostly to avoiding gaze showed larger amplitude of outcome-related ERPs than the group mostly exposed to mutual gaze. Our interpretation is that this differential neural processing contributes to the presence of different strategic patterns between the two groups of participants: those that were mostly exposed to averted gaze were more sensitive to outcomes and, in result, showed larger proportion of self-oriented win-stay-lose-shift strategy relative to those that were mostly exposed to mutual gaze.

Our study sought to examine fundamental processes related to the effects of communicative signals on social decision-making by means of implicit, objective measures. We focused on gaze, and on the effect of eye contact (i.e., mutual gaze) in particular. Like many studies (3), we chose to compare mutual gaze to a lateral, averted gaze, which was particularly suitable for our task setting (29). Although a comparison with a more "neutral" gaze would theoretically be ideal, it is important to note that in an interactive setting like the one we employed, there is no purely "neutral" gaze condition. Any gaze behavior (averted, directed to the task space, etc.) can be interpreted socially and can carry a communicative intent. Thus, rather than aiming to compare mutual gaze with a purely neutral gaze, we sought to compare mutual gaze (i.e. eye contact) with an averted gaze (i.e. no eye contact), a condition that is often used in related studies (3).

Furthermore, although real-life interactions are more complex than those occurring in our human-robot strategic game, our design captures three key aspects of social interactions: 1) two agents exchanges social signals in real-time, 2) they act upon the same, simplified, yet shared, environment, and 3) the outcome of their decision depend on the other's decision. Thus, to succeed, players have to mentalize and reason about their opponent. Therefore, we expect our findings to be relevant (and potentially generalize) to more complex, real-life contexts where dyadic interactions (either human-human or human-robot) combine communicative gaze and social decision-making. Studies of eye contact effects often rely on uni-directional settings where participants respond to gaze stimuli without affecting the other agent's actions $(3,4,49,50)$. Our design offers a unique opportunity to study these effects in the context of bi-directional interactions. Moreover, previous gaze studies in human-robot interaction made extensive use of subjective reports to examine participants' experience (15-22). Yet, little is known 
about more implicit effects of eye contact on behavioral and neural responses of which participants may not be aware. By examining implicit, objective measures, our study offers a complementary perspective and opens the avenue for future research, possibly combining different types of measures. For example, our design could be adjusted to make it suitable for measures of arousal (e.g., Galvanic skin response) and to include subjective reports of participants(e.g., self-awareness, gaze perception) in order to bridge subjective experience with underlying processes. Thereby, our design could help to extend existing theoretical accounts of eye contact effects (49) to dynamic interactions involving an active participation of both agents.

In summary, our results suggest that mutual gaze engages brain resources for managing social signals, which may or may not be relevant for the decisions to be made. In our experiment the robot gaze modulated response times, decision threshold, neural synchronization, as well as choice strategies and sensitivity to outcomes. Thus, two mechanical cameras were treated as a social signal, thereby providing striking evidence for the flexibility of human socio-cognitive mechanisms. As we build increasingly complex machines, it is therefore crucial that we endow them with adequate communicative behaviors.

\section{Materials and Methods}

\section{Participants}

43 participants were recruited for the main experiment, 3 were excluded due to high number of missing trials, excessive EEG artifacts, or technical issues during EEG recording. In total, data sets of 40 participants (mean age $=24.53 \pm 4.5,23$ women, 5 left-handed) were analyzed for the main experiment, 18 participants (mean age $=30.38 \pm 4.77,10$ women, 4 left-handed) for the first pilot study and 8 participants (mean age $=31 \pm 4.87,5$ women) for the second pilot study. No statistical methods were used to predetermine sample sizes, but our sample sizes are comparable to similar previous studies. All participants were healthy and had normal or corrected-to normal vision. No participant who took part in the pilot study was recruited also for the main experiment, meaning that each participant took part only in one of the experiments. Participants were debriefed about the purpose of the study at the end of the experiment. The experiments were performed at the Istituto Italiano di Tecnologia (IIT). Participants who took part in the pilot studies were employed by IIT. Only external participants (participants of the main experiment) received honorarium (30 €) for their participation. All experiments were conducted in accordance with the ethical standards laid down in the 2013 Declaration of Helsinki and were approved by the local ethical committee (Comitato Etico Regione Liguria). All participants provided written informed consent prior to participation. Data were stored and analyzed anonymously.

\section{Stimuli and apparatus}

Task: The experiments were carried out in a noise-attenuated room. Participants were seated facing the iCub robot at opposite sides of a table at a distance of $130 \mathrm{~cm}$. iCub is a humanoid robot(51), with 3 degrees of freedom in the eyes (common tilt, vergence, and version) and three additional degrees of freedom in the neck (roll, pitch, yaw). In our experiments, iCub was mounted on a supporting frame 
such that its eyes were at $124 \mathrm{~cm}$ from the floor which was estimated to be aligned with participants' eyes. A 24-inch LCD screen was placed horizontally on the table such that both players (participant + iCub) could see the stimuli being displayed. The stimuli consisted of a series of animations describing the events occurring during the adapted Chicken game (see "Implementation of the Human-robot Chicken game”). Before the players had to decide about their next move, we manipulated iCub's gaze to either make eye contact with the participant (mutual gaze) or avoid eye contact (averted gaze) by looking to the right or left of the table. Importantly, the target points to look at, in both the mutual and the averted gaze conditions, have been selected in order to guarantee comparable conditions in terms of joints trajectories and overall robot motion. During the rest of the trial, the robot was looking at the screen (neutral gaze), gazing at different fixation points randomly generated within the same field of view.

iCub behavior: The robot behavior was programmed using the YARP (Yet Another Robot Platform Python wrappers(52). We used iKinGazeCtrl, a 6-DOF gaze controller, to control iCub neck and eyes(53). Specifically, Azimuth, Elevation and Vergence were provided to the controller in order to make iCub look at the desired 3D Cartesian coordinates (see Table S3). In addition, gaze shifts in head movements were embedded in order to make the gaze more naturalistic. The button press was controlled using a position controller, specifically YARP IPositionControl. The facial expression (always neutral in our experiment) and lip movements were controlled using the iCub faceExpressions application. Robot speech on the other hand simply consisted of audio files played on the main workstation with external speaker placed below the robot. The audio files were recorded from female human voices and edited using the free open-source Audacity software to increase the pitch. A custommade software programmed in Python 3 and running on a with Ubuntu 20.04 LTS operating system was used to control iCub behavior, stimulus presentation, and data collection. Both players selected their actions by pressing switch buttons connected to a custom response box designed for converting input signals into regular keyboard key presses.

EEG apparatus: EEG was recorded from 64 electrode sites of an active electrode system using Ag$\mathrm{AgCl}$ electrodes, at a sampling rate of $1 \mathrm{kHz}$ (ActiCap, Brain Products, GmbH, Munich, Germany). FT9 and FT10 electrodes were displaced to F9 and F10 electrode positions to capture the horizontal ocular movements. All electrodes were referenced to FCz and re-referenced offline to average of all electrodes. Electrode impedances were kept below $10 \mathrm{k} \Omega$ throughout the experimental procedure.

\section{Procedure}

Upon EEG head preparation and after receiving the task instructions, participants started a practice session to make sure that they were performing the task properly. The main experiment lasted approximately 55 min. Participants completed 250 trials divided in 5 blocks. Between-block breaks lasted 90 seconds after the first, second and fourth block, whereas participants could take a longer break after the third block and decide when they wanted to resume the experiment. This experiment consisted of two between-participants conditions: 70\% Mutual (20 participants) or 70\% Averted (20 participants). For example, participants in the $70 \%$ Mutual condition experienced the mutual gaze in $70 \%$ of the trials and the averted gaze in the remaining $30 \%$ of each block. The sequence of trials was pseudo-randomized and participants in the 70\% Averted condition experienced the exact opposite 
sequence. The direction of the averted gaze (left or right) was counterbalanced across two subgroups in each condition. Put differently, participants were assigned to one of the following four subgroups: 70\% Mutual with averted gaze to the right, 70\% Mutual with averted gaze to the left, $70 \%$ Averted with averted gaze to the right, $70 \%$ Averted with averted gaze to the left.

Prior to the main experiment, we collected resting state EEG data with eyes closed and eyes open. After all experiments were over, participants were also asked to answer two questionnaires: one custom-made questionnaire related to self and robot's strategy, and a competitiveness index questionnaire(54). Informal discussions after the experiment confirmed that all participants noticed the gaze manipulation. The resting state and the competitiveness index questionnaire were aimed at targeting possible individual differences, which is out of the scope of this paper. Therefore, these data are not reported here.

The procedure for the pilot studies is detailed in Supplementary Materials.

\section{Drift diffusion model}

The drift diffusion model describes the intra-trial decision process in two-alternative forced choice tasks as the relative accumulation of evidence over time(32). The main parameters include the drift rate $v$ (rate of evidence accumulation), decision boundary a (threshold to be reached to make a decision) and non-decision time $t$ (dedicated to stimulus encoding or motor execution, for example). To fit the model to our data, we used Bayesian parameter estimation $(55,56)$ (see Supplementary Materials and Methods for details about the procedure).

\section{Value-based decision-making models}

This family of computational models aims to describe the inter-trial learning process underlying decision-making. In this framework, choices are made based on the available options' values, which are updated trial by trial. In this paper, we used this type of models to study the mechanisms on which participants relied during the game. Specifically, the degree to which participants reasoned about the robot's action. To do so, we readapted three computational models taken from the literature $(41,42)$. Model 1 is a reinforcement learning where most recently rewarded actions are selected and involves no reasoning about the opponent. Model 2 estimates the probabilities of opponent's actions based on recently selected choices then constructs its action values by combining the estimated probabilities with expected outcomes from the payoff matrix. Model 3 adds another level of reasoning about the opponent by integrating the influence of its own recent choices on the opponent's decisions. We optimized the models' hyperparameters to fit each participant's sequence of choices by maximizing the log-likelihood (see Supplementary Materials and Methods for details about models' implementation and the fitting procedure).

\section{EEG data analysis}

EEG data were analyzed using MATLAB® version R2018b (The Mathworks Inc., 2018), the EEGLAB(57), FieldTrip toolboxes(58) and customized scripts. The data were down-sampled to 250 $\mathrm{Hz}$, while a band-pass filter $(0.5-100 \mathrm{~Hz})$ and a notch filter $(50 \mathrm{~Hz})$ were applied. The signal was rereferenced to the common average of all electrodes. Data from one participant (from the 70\% Averted 
group) were excluded only from EEG analyses due to a large number of noisy electrodes (> 10), resulting in $\mathrm{N}=39$ for EEG analyses.

Time-frequency representations (TFRs) of oscillatory power changes were computed during the decision period, including the fixation cross and the gaze plus response period. Then, TFRs were separated for each condition (mutual gaze, averted gaze) for the abovementioned period and averaged across trials. Subsequently, the statistical analysis was performed on the epoch of interest, i.e. between the establishment of mutual/averted gaze and participants' average response time. Data were averaged to calculate power within theta (4-7) and alpha (8-12) frequency bands. In each frequency range, spatio-temporal data across conditions were compared by performing a non-parametric cluster-based permutation analyses (using a Monte-Carlo method based on paired t-statistics)(59). A full description of the time-frequency analysis is provided in Supplementary material, as well as the details of the exploratory analysis of EEG activity related to performance monitoring

\section{Statistical analysis}

Details about the statistical tests were reported in the main text. Error bars in figures indicate 95\% confidence intervals. For one-factor analyses, parametric statistical tests were used when data followed a normal distribution (Shapiro test with $\mathrm{p}>0.05$ ) and non-parametric tests when they did not. As parametric tests, we used t-test when comparing two groups or ANOVA when more. As nonparametric tests, we used Mann-Whitney test when comparing two independent groups, Wilcoxon test when comparing two paired groups and Kruskal-Wallis test when comparing more than two groups. These tests were applied using the scipy.stats Python module. They were all two-sided except MannWhitney. Two-factor analyses were performed using the JASP software. In all statistical tests, p > 0.05 was considered to be statistically non-significant.

\section{Supplementary materials}

Supplementary Materials and Methods (text)

Fig. S1: Participants performance and response time in the pilot study

Fig. S2: Evaluation of hyperparameter optimization for the value-based decision-making models

Fig. S3: Patterns of self-oriented and other-oriented strategic behaviors in participants' choice sequences

Table S1: iCub’s verbal utterances in English (pilot study) and Italian (main experiment)

Table S2: Detailed description of the player's decisions as predicted by the win-stay-lose-shift (WSLS) and counter-win-stay-lose-shift (C-WSLS) strategies

Table S3: Coordinates of robot gaze. 


\section{References and notes}

1. C. L. Kleinke, Gaze and eye contact: a research review. Psychol. Bull. 100, 78-100 (1986).

2. N. J. Emery, The eyes have it: The neuroethology, function and evolution of social gaze. Neurosci. Biobehav. Rev. 24 (2000), pp. 581-604.

3. J. K. Hietanen, Affective eye contact: An integrative review. Front. Psychol. 9 (2018), p. 1587.

4. A. F. C. Hamilton, Gazing at me: the importance of social meaning in understanding direct-gaze cues. Phil. Trans. R. Soc. B. 371 (2016), doi:DOI: 10.1098/rstb.2015.0080.

5. A. Senju, M. Johnson, The eye contact effect: mechanisms and development. Trends Cogn. Sci. 13, 127-134 (2009).

6. J. K. Rilling, A. G. Sanfey, The Neuroscience of Social Decision-Making. Annu. Rev. Psychol. 62, 23-48 (2011).

7. Y. Wang, D. M. Kuhlman, K. Roberts, B. Yuan, Z. Zhang, W. Zhang, R. F. Simons, Social value orientation modulates the FRN and P300 in the chicken game. Biol. Psychol. 127, 89-98 (2017).

8. Y. Chen, J. Lu, Y. Wang, Z. Feng, B. Yuan, Social distance influences the outcome evaluation of cooperation and conflict: Evidence from event-related potentials. Neurosci. Lett. 647, 78-84 (2017).

9. D. E. Asher, A. Zaldivar, B. Barton, A. A. Brewer, J. L. Krichmar, Reciprocity and retaliation in social games with adaptive agents. IEEE Trans. Auton. Ment. Dev. 4, 226-238 (2012).

10. E. Benítez Sandoval, J. Brandstetter, M. Obaid, C. Bartneck, Reciprocity in Human-Robot Interaction: A Quantitative Approach Through the Prisoner's Dilemma and the Ultimatum Game. Int. J. Soc. Robot. 8, 303-317 (2016).

11. T. Chaminade, D. Rosset, D. Da Fonseca, B. Nazarian, E. Lutcher, G. Cheng, C. Deruelle, How do we think machines think? An fMRI study of alleged competition with an artificial intelligence. Front. Hum. Neurosci. 6, 103 (2012).

12. H. Takahashi, K. Terada, T. Morita, S. Suzuki, T. Haji, H. Kozima, M. Yoshikawa, Y. Matsumoto, T. Omori, M. Asada, E. Naito, Different impressions of other agents obtained through social interaction uniquely modulate dorsal and ventral pathway activities in the social human brain. Cortex. 58, 289-300 (2014).

13. T. Hsieh, B. Chaudhury, E. Cross, Human-robot cooperation in prisoner dilemma games people behave more reciprocally than prosocially toward robots, in ACM/IEEE International Conference on Human-Robot Interaction (IEEE Computer Society, New York, NY, USA, 2020; https://dl.acm.org/doi/10.1145/3371382.3378309), pp. 257-259.

14. M. Fraune, S. Sherrin, S. Šabanović, E. R. Smith, Is human-robot interaction more competitive between groups than between individuals?, in 2019 14th ACM/IEEE International Conference on Human-Robot Interaction (HRI) (2019), pp. 104-113.

15. A. Moon, M. Zheng, D. M. Troniak, B. A. Blumer, B. Gleeson, K. MacLean, M. K. X. J. Pan, E. A. Croft, Meet me where i'm gazing: How shared attention gaze affects human-robot handover timing, in ACM/IEEE International Conference on Human-Robot Interaction (IEEE Computer Society, New York, NY, USA, 2014; https://dl.acm.org/doi/10.1145/2559636.2559656), pp. 334-341. 
16. T. Yonezawa, H. Yamazoe, A. Utsumi, S. Abe, Gaze-communicative behavior of stuffed-toy robot with joint attention and eye contact based on ambient gaze-tracking, in Proceedings of the 9th international conference on Multimodal interfaces (2007), pp. 140-145.

17. K. Kompatsiari, F. Ciardo, V. Tikhanoff, G. Metta, · Agnieszka Wykowska, It's in the Eyes: The Engaging Role of Eye Contact in HRI. Int. J. Soc. Robot. (2019), doi:10.1007/s12369-01900565-4.

18. M. Imai, T. Kanda, T. Ono, H. Ishiguro, K. Mase, Robot mediated round table: Analysis of the effect of robot's gaze, in Proceedings - IEEE International Workshop on Robot and Human Interactive Communication (2002; https://keio.pure.elsevier.com/en/publications/robotmediated-round-table-analysis-of-the-effect-of-robots-gaze), pp. 411-416.

19. A. Ito, S. Hayakawa, T. Terada, Why robots need body for mind communication-an attempt of eye-contact between human and robot, in Robot and Human Interactive, 2004. ROMAN 2004. 13th IEEE International Workshop on (2004), pp. 473-478.

20. D. Szafir, B. Mutlu, D. Szafir, Pay Attention! Designing Adaptive Agents that Monitor and Improve User Engagement, in CHI '12: Proceedings of the SIGCHI Conference on Human Factors in Computing Systems (2012; https://www.researchgate.net/publication/235838918), pp. 11-20.

21. J. Mumm, B. Mutlu, Human-robot proxemics: Physical and psychological distancing in humanrobot interaction, in HRI 2011 - Proceedings of the 6th ACM/IEEE International Conference on Human-Robot Interaction (ACM Press, New York, New York, USA, 2011; http://portal.acm.org/citation.cfm?doid=1957656.1957786), pp. 331-338.

22. J. J. Choi, Y. Kim, S. S. Kwak, Have you ever lied?: The impacts of gaze avoidance on people's perception of a robot, in ACM/IEEE International Conference on Human-Robot Interaction (2013), pp. 105-106.

23. J.-D. Boucher, U. Pattacini, A. Lelong, G. Bailly, F. Elisei, S. Fagel, P. F. Dominey, J. VentreDominey, I Reach Faster When I See You Look: Gaze Effects in Human-Human and HumanRobot Face-to-Face Cooperation. Front. Neurorobot. 6, 3 (2012).

24. K. Kompatsiari, F. Ciardo, D. De Tommaso, A. Wykowska, Measuring engagement elicited by eye contact in Human-Robot Interaction, in IEEE International Conference on Intelligent Robots and Systems (Institute of Electrical and Electronics Engineers Inc., 2019), pp. 69796985.

25. S. Ehrlich, A. Wykowska, K. Ramirez-Amaro, G. Cheng, When to engage in interaction - And how? EEG-based enhancement of robot's ability to sense social signals in HRI, in IEEE-RAS International Conference on Humanoid Robots (IEEE Computer Society, 2015), vols. 2015February, pp. 1104-1109.

26. K. Kompatsiari, F. Bossi, A. Wykowska, Eye contact during joint attention with a humanoid robot modulates oscillatory brain activity. Soc. Cogn. Affect. Neurosci. 2021, 1-10 (2021).

27. G. Metta, L. Natale, F. Nori, G. Sandini, D. Vernon, L. Fadiga, C. von Hofsten, K. Rosander, M. Lopes, J. Santos-Victor, A. Bernardino, L. Montesano, The iCub humanoid robot: An opensystems platform for research in cognitive development. Neural Networks. 23, 1125-1134 (2010).

28. A. Rapoport, A. M. Chammah, The Game of Chicken. Am. Behav. Sci. 10, 10-28 (1966). 
29. Averted (lateral) gaze has been extensively used in literature as a comparison condition to mutual gaze, for a review see ${ }^{3}$. Critically, in the current experiment, it offers the advantage of implementing similar movement profiles for the two gaze conditions (mutual, averted) on the robot. Please note that a "neutral" no-gaze condition is not included as a baseline comparison because it does not enable the same readiness to respond, i.e., the fact that it does not include any motion compared to the other two gaze conditions could result in an additional expectancy effect that would potentially delay participants’ response times.

30. M. Nowak, K. Sigmund, A strategy of win-stay, lose-shift that outperforms tit-for-tat in the Prisoner’s Dilemma game. Nature. 364, 56-58 (1993).

31. Please note that there is not an a-priori "optimal” strategy per se in the Chicken game since the outcome depends on the opponents' decision which cannot be optimally predicted. As an "optimal” strategy, we refer to an ideal case where participants understood the robot's strategy (WSLS) and select their actions accordingly.

32. R. Ratcliff, G. McKoon, The diffusion decision model: Theory and data for two-choice decision tasks. Neural Comput. 20 (2008), pp. 873-922.

33. L. M. Ward, Synchronous neural oscillations and cognitive processes. Trends Cogn. Sci. 7, 553559 (2003).

34. J. Jacobs, G. Hwang, T. Curran, M. Kahana, EEG oscillations and recognition memory: Theta correlates of memory retrieval and decision making. Neuroimage. 32, 978-987 (2006).

35. A. Rajan, S. N. Siegel, Y. Liu, J. Bengson, G. R. Mangun, M. Ding, Theta Oscillations Index Frontal Decision-Making and Mediate Reciprocal Frontal-Parietal Interactions in Willed Attention. Cereb. Cortex. 29, 2832-2843 (2019).

36. J. J. Foxe, A. C. Snyder, The role of alpha-band brain oscillations as a sensory suppression mechanism during selective attention. Front. Psychol. 2, 154 (2011).

37. A. Gale, B. Lucas, R. Nissim, B. Harpham, Some EEG correlates of face-to-face contact. Br. J. Soc. Clin. Psychol. 11, 326-332 (1972).

38. J. Hietanen, J. Leppänen, M. Peltola, K. Linna-aho, H. Ruuhiala, Seeing direct and averted gaze activates the approach-avoidance motivational brain systems. Neuropsychologia. 46, 2423-2430 (2008).

39. O. Jensen, J. Gelfand, J. Kounios, J. Lisman, Oscillations in the alpha band (9-12 Hz) increase with memory load during retention in a short-term memory task. Cereb. Cortex. 12, 877-882 (2002).

40. N. R. Cooper, R. J. Croft, S. J. J. Dominey, A. P. Burgess, J. H. Gruzelier, Paradox lost? Exploring the role of alpha oscillations during externally vs. internally directed attention and the implications for idling and inhibition hypotheses. Int. J. Psychophysiol. 47, 65-74 (2003).

41. A. N. Hampton, P. Bossaerts, J. P. O’Doherty, Neural correlates of mentalizing-related computations during strategic interactions in humans. Natl. Acad Sci. 105, 6741-6746 (2008).

42. R. Rescorla, A. Wagner, A theory of Pavlovian conditioning: Variations in the effectiveness of reinforcement and nonreinforcement. Class. Cond. ii. 64 (available at https://ci.nii.ac.jp/naid/10024275851/).

43. R. San Martín, Event-related potential studies of outcome processing and feedback-guided learning. Front. Hum. Neurosci., 1-40 (2012). 
44. W. Gehring, Y. Liu, J. Orr, J. Carp, The error-related negativity (ERN/Ne). (2012) (available at https://psycnet.apa.org/record/2013-01016-010).

45. D. Polezzi, L. Lotto, I. Daum, G. Sartori, R. Rumiati, Predicting outcomes of decisions in the brain. Behav. Brain Res. 187, 116-122 (2008).

46. C. Holroyd, M. Coles, The neural basis of human error processing: Reinforcement learning, dopamine, and the error-related negativity. Psychol. Rev. 109, 679-709 (2002).

47. A. Abubshait, P. J. Beatty, C. G. McDonald, C. D. Hassall, O. E. Krigolson, E. Wiese, A winwin situation: Does familiarity with a social robot modulate feedback monitoring and learning? Cogn. Affect. Behav. Neurosci. (2021), doi:10.3758/s13415-021-00895-9.

48. D. Polezzi, L. Lotto, I. Daum, G. Sartori, R. Rumiati, Predicting outcomes of decisions in the brain. Behav. Brain Res. 187, 116-122 (2008).

49. L. Conty, N. George, J. K. Hietanen, Watching Eyes effects: When others meet the self. Conscious. Cogn. 45 (2016), pp. 184-197.

50. M. Bateson, D. Nettle, G. Roberts, Cues of being watched enhance cooperation in a real-world setting. Biol. Lett. 2, 412-414 (2006).

51. G. Metta, L. Natale, F. Nori, G. Sandini, D. Vernon, L. Fadiga, C. von Hofsten, K. Rosander, M. Lopes, J. Santos-Victor, A. Bernardino, L. Montesano, The iCub humanoid robot: An opensystems platform for research in cognitive development. Neural Networks. 23, 1125-1134 (2010).

52. G. Metta, P. Fitzpatrick, L. Natale, YARP: Yet Another Robot Platform. Int. J. Adv. Robot. Syst. 3, 8 (2006).

53. A. Roncone, U. Pattacini, G. Metta, L. Natale, A Cartesian 6-DoF Gaze Controller for Humanoid Robots, in Robotics: science and systems (http://wiki.icub.org/iCub/main/).

54. J. Houston, J. Kinnie, B. Lupo, C. Terry, S. Ho, Competitiveness and conflict behavior in simulation of a social dilemma. Psychol. Rep. 86, 1219-1225 (2000).

55. T. V. Wiecki, I. Sofer, M. J. Frank, HDDM: Hierarchical Bayesian estimation of the DriftDiffusion Model in Python. Front. Neuroinform. 7, 14 (2013).

56. J. K. Kruschke, Bayesian estimation supersedes the t test. J. Exp. Psychol. Gen. 142, 573-603 (2013).

57. A. Delorme, S. Makeig, EEGLAB: An open source toolbox for analysis of single-trial EEG dynamics including independent component analysis. J. Neurosci. Methods. 134, 9-21 (2004).

58. R. Oostenveld, P. Fries, E. Maris, J. M. Schoffelen, FieldTrip: Open source software for advanced analysis of MEG, EEG, and invasive electrophysiological datComput. Intell. Neurosci. 2011 (2011), doi:10.1155/2011/156869.

59. E. Maris, R. Oostenveld, Nonparametric statistical testing of EEG- and MEG-data. J. Neurosci. Methods. 164, 177-190 (2007). 
Acknowledgments: The authors would like to thank Abdulaziz Abubshait, Francesco Bossi, Francesca Ciardo, Jairo Perez-Osorio and Cesco Willemse for their advice and feedback, Adam Wojciech Łukomski for his help in developing the experiment software, and Marco Crepaldi for his work on the response box controller. Funding: This project has received funding from the European Research Council (ERC) under the European Union's Horizon 2020 research and innovation program (grant awarded to A.W., titled “InStance: Intentional stance for social attunement.” G.A. no.: ERC-2016-StG715058). The content of this paper is the sole responsibility of the authors. The European Commission or its services cannot be held responsible for any use that may be made of the information it contains. Author contributions: M.B., K.K. and A.W. designed the study. M.B. and D.D.T. programmed the experiment. M.B., K.K. and I.Z. collected the data. M.B. and K.K. analyzed the data. M.B., K.K. and A.W. wrote the manuscript. Competing interests: The authors declare no competing interest. Data and materials availability: All data needed to evaluate the conclusions in the paper are present in the paper or the Supplementary Materials. The data for this study are available in the OSF database https:// osf.io/wj5kq/. 